\title{
An evidence-based review of obatoclax mesylate in the treatment of hematological malignancies
}

This article was published in the following Dove Press journal:

Core Evidence

13 March 2013

Number of times this article has been viewed

\section{Carolyn A Goard \\ Aaron D Schimmer \\ Princess Margaret Cancer Centre, Ontario Cancer Institute, University Health Network, Toronto, ON, Canada}

Correspondence: Aaron D Schimmer 610 University Avenue, Room 9-605, Toronto, ON, Canada M5G 2M9

$\mathrm{Tel}+\mathrm{I} 4169462838$

Fax + I 4169466546

Email aaron.schimmer@utoronto.ca Ontario Cancer Institute,

Abstract: Obatoclax mesylate is an intravenously-administered drug under investigation in Phase I and II clinical trials as a novel anticancer therapeutic for hematological malignancies and solid tumors. Obatoclax was developed as a pan-inhibitor of antiapoptotic members of the B cell chronic lymphocytic leukemia/lymphoma 2 (BCL-2) family of proteins, which control the intrinsic or mitochondrial pathway of apoptosis. Resistance to apoptosis through dysregulation of BCL-2 family members is commonly observed in hematological malignancies, and can be linked to therapeutic resistance and poor clinical outcomes. By inhibiting pro-survival BCL-2 family proteins, including MCL-1, obatoclax is proposed to (1) trigger cell death as a single agent, and (2) potentiate the anticancer effects of other therapeutics. Preclinical investigations have supported these proposals and have provided evidence suggestive of a promising therapeutic index for this drug. Phase I trials of obatoclax mesylate in leukemia and lymphoma have defined well-tolerated regimens and have identified transient neurotoxicity as the most common adverse effect of this drug. In these studies, a limited number of objective responses were observed, along with hematological improvement in a larger proportion of treated patients. Published Phase II evaluations in lymphoma and myelofibrosis, however, have not reported robust single-agent activity. Emerging evidence from ongoing preclinical and clinical investigations suggests that the full potential of obatoclax mesylate as a novel anticancer agent may be realized (1) in rational combination treatments, and (2) when guided by molecular predictors of therapeutic response. By understanding the molecular underpinnings of obatoclax response, along with optimal therapeutic regimens and indications, the potential of obatoclax mesylate for the treatment of hematological malignancies may be further clarified.

Keywords: obatoclax, leukemia, lymphoma, myelofibrosis, BCL-2, BH3 mimetic

Core evidence clinical impact summary for obatoclax mesylate in hematological malignancies

\begin{tabular}{|lll|}
\hline Outcome measure & Evidence & Implications \\
\hline $\begin{array}{l}\text { Disease-oriented evidence } \\
\text { Induces cell death in }\end{array}$ & $\begin{array}{l}\text { Cell death was induced in cell lines and } \\
\text { ex vivo primary samples from hematological } \\
\text { cancers, through BAK/BAX-dependent } \\
\text { and independent effects }\end{array}$ & $\begin{array}{l}\text { Obatoclax may act } \\
\text { through BH3 mimetic- } \\
\text { dependent and } \\
\text { independent activities on }\end{array}$ \\
$\begin{array}{lll}\text { Induces correlates of } \\
\text { apoptosis in patients }\end{array}$ & $\begin{array}{l}\text { Phase I trial in chronic lymphocytic } \\
\text { leukemia observed increases in molecular } \\
\text { correlates of apoptosis in some, but not } \\
\text { all patients, and these were not always }\end{array}$ & $\begin{array}{l}\text { Obatoclax appears to } \\
\text { have "on-target" biological } \\
\text { effects in some patients } \\
\end{array}$ \\
& seen in responding patients & with hematological \\
\end{tabular}




\begin{tabular}{|c|c|c|}
\hline $\begin{array}{l}\text { (Continued) } \\
\text { Outcome measure }\end{array}$ & Evidence & Implications \\
\hline \multicolumn{3}{|c|}{ Patient-oriented evidence } \\
\hline $\begin{array}{l}\text { Recommended doses } \\
\text { and schedules defined }\end{array}$ & $\begin{array}{l}\text { Phase I trials have defined well- or } \\
\text { maximally tolerated doses and schedules }\end{array}$ & $\begin{array}{l}\text { Recommended doses } \\
\text { and schedules can be } \\
\text { evaluated in ongoing and } \\
\text { subsequent indication- } \\
\text { specific Phase II trials }\end{array}$ \\
\hline $\begin{array}{l}\text { Generally well } \\
\text { tolerated }\end{array}$ & $\begin{array}{l}\text { Safety and adverse event profiles } \\
\text { have been defined by Phase I trials }\end{array}$ & $\begin{array}{l}\text { Common neurological } \\
\text { toxicities are transient } \\
\text { and manageable under } \\
\text { recommended treatment } \\
\text { regimens }\end{array}$ \\
\hline $\begin{array}{l}\text { Exhibits clinical } \\
\text { activity alone }\end{array}$ & $\begin{array}{l}\text { Phase I trials observed objective responses } \\
\text { in I acute myelogenous leukemia, I } \\
\text { chronic lymphocytic leukemia, and } \\
\text { I lymphoma patient (each approximately } 4 \% \\
\text { of treated patients) Phase I trials observed } \\
\text { hematological improvement in approximately } \\
20 \% \text { to } 30 \% \text { of treated patients } \\
\text { Phase II trials in myelofibrosis or Hodgkin } \\
\text { lymphoma did not observe objective } \\
\text { responses }\end{array}$ & $\begin{array}{l}\text { As a single agent, clinical } \\
\text { activity may be limited } \\
\text { to a small subset of } \\
\text { treated patients, pending } \\
\text { availability of indication- } \\
\text { specific Phase II trial data }\end{array}$ \\
\hline $\begin{array}{l}\text { Exhibits clinical } \\
\text { activity in combination }\end{array}$ & $\begin{array}{l}\text { Phase I trials combining obatoclax } \\
\text { mesylate with other therapeutics have } \\
\text { provided early evidence of promising } \\
\text { response rates (approximately } \\
25 \% \text { to } 50 \% \text { ) }\end{array}$ & $\begin{array}{l}\text { The potential efficacy of } \\
\text { obatoclax mesylate in } \\
\text { combination with other } \\
\text { anticancer therapeutics } \\
\text { may warrant further } \\
\text { investigation in ongoing } \\
\text { and subsequent Phase } \\
\text { II trials }\end{array}$ \\
\hline Economic evidence & Not yet formally assessed & \\
\hline
\end{tabular}

\section{Background and scope}

Apoptosis is a form of programmed cell death essential for the removal of unneeded or damaged cells. Characterized by the morphological hallmarks of cellular shrinkage, nuclear condensation, and membrane blebbing, apoptosis is a complex, highly-regulated process classically executed by the caspase family of cysteine proteases. ${ }^{1,2}$ The "intrinsic," or mitochondrial, pathway of apoptosis can be induced upon a variety of cellular stresses, culminating in mitochondrial outer membrane permeabilization; release of cytochrome c and other mitochondrial proteins into the cytoplasm; and activation of downstream effector caspases., ${ }^{2,3}$ Regulation of the pivotal point of mitochondrial outer membrane permeabilization is mediated in a large part by the $\mathrm{B}$ cell chronic lymphocytic leukemia/lymphoma 2 (BCL-2) protein family. ${ }^{4}$ Here, we provide an evidence-based discussion of the clinical development and potential of one novel apoptosis modulator, the small molecule BCL-2 inhibitor obatoclax mesylate (also known as GX15-070; originally developed by Gemin X, Montreal, QC, Canada; acquired by Teva, Israel).
BCL-2 was discovered almost three decades ago in efforts to identify genes involved in the $\mathrm{t}(14 ; 18)$ translocation commonly observed in B-cell malignancies. ${ }^{5,6} \mathrm{BCL}-2$ became the first oncogene demonstrated to act through the promotion of cell survival rather than proliferation..$^{7-9}$ Subsequently, several related pro- and antiapoptotic family members were identified based on shared BCL-2 homology (BH) domains (Table 1, Figure 1). ${ }^{4,10}$ BCL-2 and related antiapoptotic family members protect cells against apoptosis by (1) heterodimerizing and sequestering the proapoptotic BCL-2 family members BAX and BAK or their activators, (2) stabilizing the outer mitochondrial membrane, and (3) modulating intracellular calcium flux. For a complete review of BCL-2 family function in mitochondrial apoptosis, see Kroemer et $\mathrm{al}^{1}$ and Youle and Strasser. ${ }^{10}$ Cells also possess endogenous inhibitors of BCL-2 and related antiapoptotic family members, the BH3only proteins. ${ }^{4}$ These proteins sense apoptotic stimuli and act as activators and/or sensitizers in the process of BAX- and BAK-mediated permeabilization of the outer mitochondrial membrane. BH3-only proteins can bind to antiapoptotic BCL-2 members, allowing displacement of bound BAX 
Table I BCL-2 family members involved in apoptosis

\begin{tabular}{|c|c|c|}
\hline \multirow[t]{2}{*}{ Classification } & \multicolumn{2}{|c|}{ Family members } \\
\hline & Symbol & Name \\
\hline \multirow{6}{*}{$\begin{array}{l}\text { Multidomain } \\
\text { antiapoptotic }\end{array}$} & BCL-2 & B-cell CLL/lymphoma 2 \\
\hline & $B C L-X L$ & $\begin{array}{l}\text { BCL extra long; also known as } \\
\text { BCL-2-like I }\end{array}$ \\
\hline & BCL-w & $\begin{array}{l}\text { BCL-2-like protein 2; also known as } \\
\text { BCL-2-like } 2\end{array}$ \\
\hline & BCL-B & BCL-2-like 10 (apoptosis facilitator) \\
\hline & BFL-I & $\begin{array}{l}\mathrm{BCL}-2 \text {-related gene expression in fetal liver; } \\
\text { also known as } \mathrm{BCL2} \text {-related protein } \mathrm{Al}\end{array}$ \\
\hline & MCL-I & $\begin{array}{l}\text { myeloid cell leukemia I; also known } \\
\text { as myeloid cell leukemia sequence I } \\
\text { (BCL2-related) }\end{array}$ \\
\hline Multidomain & BAX & BCL-2-associated $X$ protein \\
\hline proapoptotic & BAK & BCL-2-antagonist/killer I \\
\hline \multirow[t]{2}{*}{$\begin{array}{l}\mathrm{BH} \text {-only } \\
\text { activator }\end{array}$} & BIM & $\begin{array}{l}\text { BCL-2 interacting mediator of cell death; } \\
\text { also known as BCL-2-like II } \\
\text { (apoptosis facilitator) }\end{array}$ \\
\hline & BID & $\mathrm{BH} 3$ interacting domain death agonist \\
\hline BH3-only & BAD & BCL2-associated agonist of cell death \\
\hline \multirow[t]{5}{*}{ sensitizer } & BIK & $\begin{array}{l}\text { BCL2-interacting killer } \\
\text { (apoptosis-inducing) }\end{array}$ \\
\hline & BMF & BCL-2 modifying factor \\
\hline & HRK & $\begin{array}{l}\text { harakiri, } \mathrm{BCL}-2 \text { interacting protein } \\
\text { (contains only } \mathrm{BH} 3 \text { domain) }\end{array}$ \\
\hline & NOXA & $\begin{array}{l}\text { protein noxa; also known as phorbol-12- } \\
\text { myristate-13-acetate-induced protein I }\end{array}$ \\
\hline & PUMA & $\begin{array}{l}\text { p53 upregulated modulator of apoptosis; } \\
\text { also known as BCL-2 binding component } 3\end{array}$ \\
\hline
\end{tabular}

Note: Classifications adapted from Davids and Letai. ${ }^{33}$

Abbreviations: $B C L, B$ cell CLL/lymphoma 2; CLL chronic lymphocytic leukemia; $\mathrm{BH} 3, \mathrm{BCL}-2$ homology domain 3.

or BAK and/or BH3-only proteins with activator functions (Figure 1A).

Elevated expression of BCL-2 and related antiapoptotic family members often occurs in hematological malignancies as a result of translocation, gene amplification, or mechanisms independent of genomic aberrations (Figure 1B). ${ }^{11}$ Overexpression of these proteins, or an alteration in the balance of expression and binding of pro- and antiapoptotic family members, can be associated with chemoresistance and poor clinical outcomes, suggesting that this protein family may be an attractive therapeutic target. ${ }^{12-18}$ Moreover, given the integrity of apoptotic regulation in normal cells, targeting dysregulated BCL-2 family activity in tumor cells may offer a promising therapeutic index. ${ }^{13}$

\section{Therapeutic targeting of antiapoptotic BCL-2 family members}

Early proof-of-principle for the therapeutic targeting of BCL-2 and its related antiapoptotic family members was provided by the development of a modified peptide derived from the

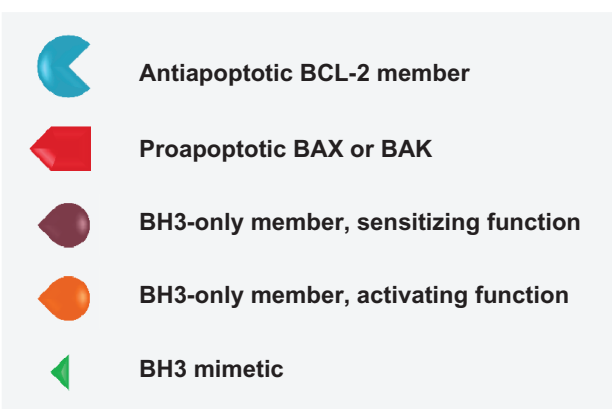

A Normal cell

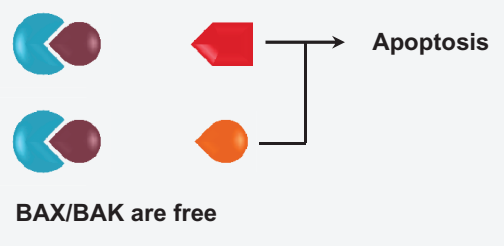

B Tumor cell

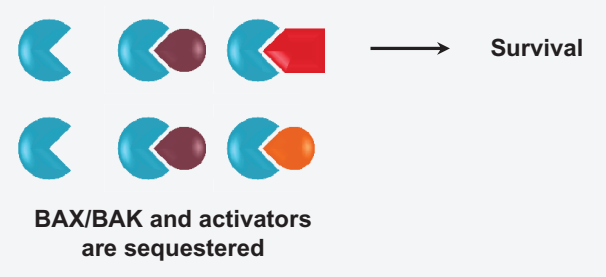

C Tumor cell treated with $\mathrm{BH} 3$ mimetic

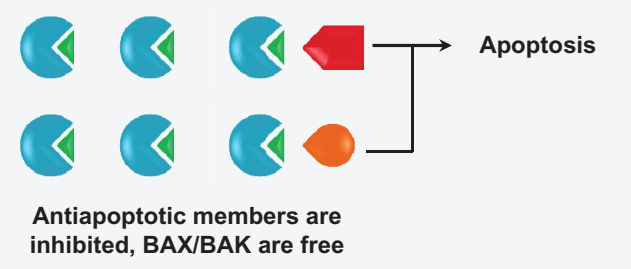

Figure I Interactions between $\mathrm{BCL}-2$ family members and $\mathrm{BH} 3$ mimetics. (A) Under apoptotic conditions in normal cells, free and activated BAX/BAK can undergo a conformational change and oligomerize in the outer mitochondrial membrane, inducing permeabilization and subsequent apoptosis. $\mathrm{BH}$-only proteins sense apoptotic stimuli and modulate the interplay between pro- and antiapoptotic multidomain family members. BH3-only proteins may act as (I) direct activators of BAX/BAK oligomerization, if they overcome inhibition by antiapoptotic family members; (2) sensitizers, if their binding to antiapoptotic members frees direct activator BH3-only proteins; and/or (3) indirect activators, if their binding to antiapoptotic family members frees BAX/BAK. (B) Many hematological tumors upregulate expression of antiapoptotic BCL-2 family members to acquire a prosurvival phenotype by functionally neutralizing proapoptotic family members. (C) Tumor cells dependent on high levels of antiapoptotic BCL-2 family members are predicted to be highly responsive to $\mathrm{BH} 3$ mimetics, which will bind antiapoptotic members, releasing both BAX/BAK and proapoptotic $\mathrm{BH} 3$-only proteins.

Notes: $\mathrm{BH} 3$ mimetics may therefore robustly induce mitochondrial outer membrane permeabilization and apoptosis, positioning them as novel anticancer therapeutic options for tumors with these characteristics.

Abbreviations: BCL-2, B-cell chronic lymphocytic leukemia/lymphoma 2; BAX, $\mathrm{BCL}$-2-associated X protein; BAK, BCL-2-antagonist/killer I; BH3, BCL-2 homology domain 3 .

BH3 domain of the BH3-only protein BID. This stabilized, cell-permeable peptide bound to BCL-2 and BCL-XL and had anticancer activity in leukemia cell lines and xenografts. ${ }^{19}$ 
Antisense oligonucleotides were the first therapeutic approach targeting BCL-2 to reach clinical trials. Oblimersen sodium (Genasense; Genta, Berkeley Heights, NJ, USA), a modified oligodeoxynucleotide complementary to BCL-2 mRNA, was developed as an antisense strategy to specifically disrupt BCL-2 expression. ${ }^{20,21}$ Despite promising signals in early-phase studies, Phase III trials in multiple myeloma, acute myelogenous leukemia (AML), chronic lymphocytic leukemia (CLL), and melanoma did not demonstrate improvement in overall survival and the drug has not yet obtained regulatory approval. ${ }^{22-25}$ The reasons underlying the lack of improvement in overall survival are complex and likely multifactorial. It is possible that the lack of efficacy reflects drawbacks of the antisense approach, rather than the value of BCL-2 as a target.

Small molecule inhibitors of BCL-2 and its antiapoptotic relatives that can occupy the $\mathrm{BH} 3$-binding cleft and prevent binding of proapoptotic BAX, BAK, or BH3-only proteins have also been developed (Figure 1C). Several small molecules, including the natural product gossypol and synthetic compounds such as ABT-737 and obatoclax, act as BH3 mimetics in vitro, demonstrating more permissive interactions with multiple antiapoptotic BCL-2 family members. ${ }^{26}$ Variants of three such small molecules have been evaluated in Phase I and II trials in cancer patients: (1) ABT-263, an orally-bioavailable derivative of ABT737 (navitoclax; Abbott Laboratories, Abbott Park, IL, USA);27 (2) AT-101, an orally-bioavailable enantiomer of gossypol (Ascenta Therapeutics, Malvern, PA, USA); ${ }^{28}$ and (3) obatoclax mesylate, a salt formulated for intravenous administration. ${ }^{29}$ These compounds differ in several respects, including their specificity for BCL-2 family members (Figure 2).

ABT-737 was rationally designed as an inhibitor of BCL-2, BCL-XL, and BCL-w through a nuclear magnetic resonance (NMR)-based structure-activity relationship (SAR) approach. ${ }^{30}$ The closely-related ABT-263 has demonstrated limited activity as a single agent in a Phase II trial in lung cancer, and subsequent combination studies are planned. ${ }^{31}$ Promising responses to ABT-263 in Phase I trials in lymphoid malignancies have supported the initiation of Phase II combination studies, which remain in progress..$^{32}$ While the oral availability of this compound is an attractive property for chronic administration, challenges to optimal therapeutic efficacy remain, including the inability of ABT-737 and ABT-263 to inhibit MCL-1 and the common incidence of thrombocytopenia as a result of potent BCL-XL inhibition in platelets. ${ }^{33,34}$ Recently,

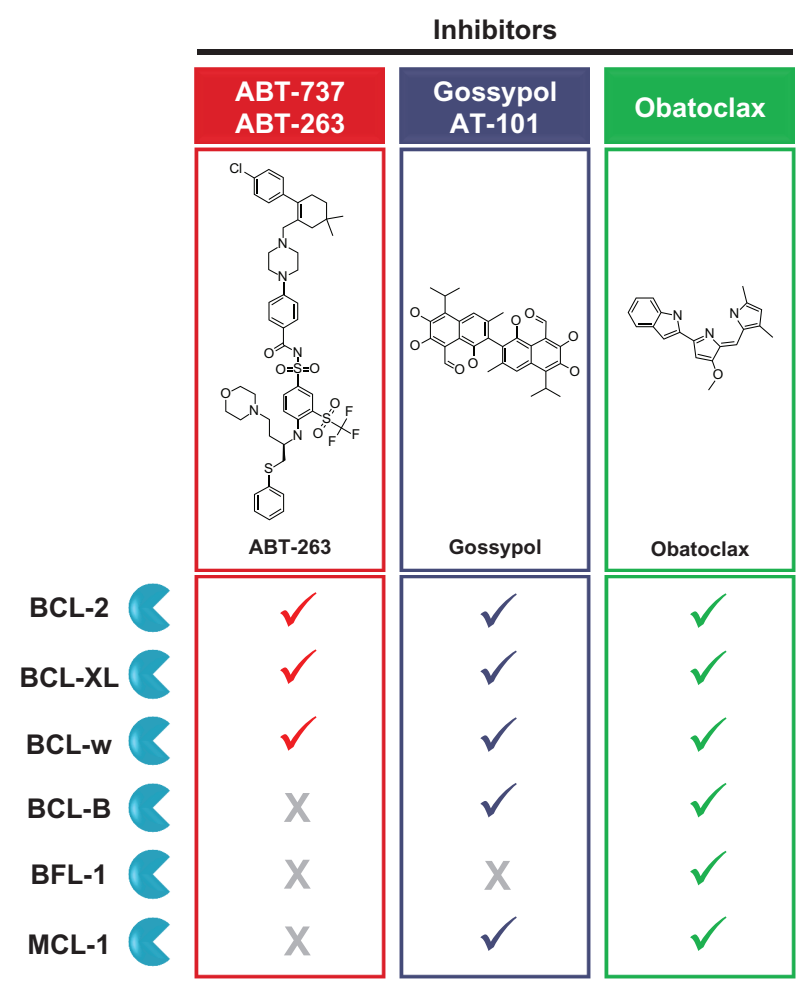

Figure 2 Binding specificity of clinically-developed $\mathrm{BH} 3$ mimetic small molecules. Notes: Checkmarks indicate small molecules that have been found to bind each antiapoptotic BCL-2 family member in vitro as discussed in the text. The ABT compounds bind three family members with high affinity. In contrast, gossypol and obatoclax act as pan-inhibitors, binding to many family members with a relatively lower affinity.

Abbreviation: $\mathrm{BH} 3, \mathrm{BCL}-2$ homology domain 3.

navitoclax has been reengineered to reduce interactions with BCL-XL and thus avoid the adverse "on-target" thrombocytopenia that limits its use in hematological cancer patients. ${ }^{35}$ The resulting compound, ABT-199, selectively inhibits BCL-2 and reduces the viability of hematological tumor cells with deregulated BCL-2 expression without substantial toxicity to platelets. Importantly, first-in-human administration of ABT-199 to three patients with refractory CLL triggered a rapid antileukemic response, while largely sparing platelets from damage. Ongoing clinical evaluation in CLL and lymphoma will further clarify the utility of ABT-199 in the treatment of BCL-2-dependent hematological malignancies. ${ }^{36}$

Gossypol was identified as a $\mathrm{BH} 3$ mimetic, binding to BCL-XL in a screen of natural compounds guided by NMR and fluorescence polarization. ${ }^{28}$ Subsequent studies identified it as a pan-BCL-2 family inhibitor, binding to most antiapoptotic family members. ${ }^{26}$ Phase II trials in prostate and lung cancers using AT-101 alone or in combination treatments have not yet demonstrated convincing clinical activity. ${ }^{37-41}$ Results from clinical evaluation of AT-101 in hematological malignancies are forthcoming. 


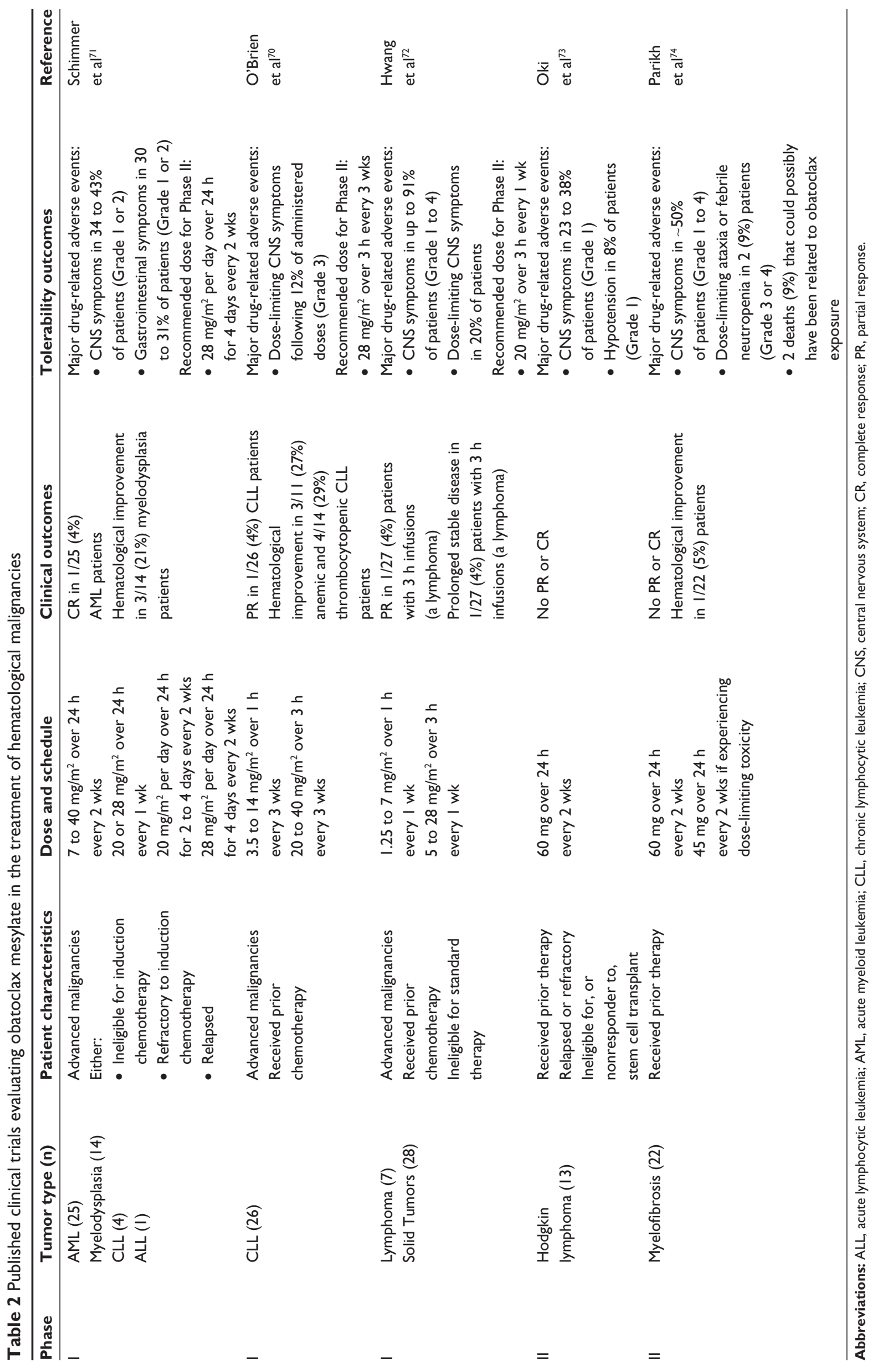


Obatoclax was similarly identified as a pan-BCL-2 family inhibitor, and has been evaluated preclinically and clinically in both hematological malignancies and solid tumors. For an overview of the evaluation of obatoclax in solid tumors, see a recent review by Joudeh and Claxton. ${ }^{42}$

\section{Preclinical efficacy of obatoclax in hematological malignancies}

Obatoclax was developed from a lead compound identified in a functional screen of natural compounds that detected disruption of protein-protein interactions between members of the BCL-2 family. ${ }^{29}$ Obatoclax and its predecessor analogs bind to BCL-2, BCL-XL, BCL-w, BCL-B, BFL-1, and MCL-1 in vitro, albeit with lower affinity than that with which the ABT compounds bind their targets. ${ }^{26,29,43}$ The ability of obatoclax to inhibit MCL-1 may be particularly important, given that several hematological malignancies appear to depend on this protein for survival, such as acute lymphoblastic leukemia (ALL) ${ }^{44} \mathrm{CLL},{ }^{44}$ multiple myeloma, ${ }^{45}$ and diffuse large B-cell lymphoma (DLBCL). ${ }^{46}$

In intact cancer cell lines, obatoclax can induce oligomerization of BAK in the mitochondria, disrupt mitochondrial function, and activate caspases. ${ }^{29}$ As a single agent, obatoclax has demonstrated anticancer activity in cell lines and/or primary cells isolated from AML, ${ }^{47}$ mast cell leukemia and systemic mastocytosis, ${ }^{48,49} \mathrm{ALL},{ }^{50,51} \mathrm{CLL},{ }^{52-55}$ lymphoma, ${ }^{56,57}$ and multiple myeloma. ${ }^{58}$ These activities have included not only induction of cell death, but also cell cycle arrest, ${ }^{47}$ reduced colony formation or clonogenicity, ${ }^{43,47}$ and death-promoting autophagy. ${ }^{51,56}$ Many studies of obatoclax activity have observed evidence consistent with a BH3 mimetic induction of apoptosis. . $3,47,51,54,57,58$ However, our understanding of the biological mechanisms underlying its spectrum of anticancer activities is still evolving. For instance, obatoclax-induced cytotoxicity is still observed in some cell systems lacking BAK and BAX expression, suggesting the existence of complementary activities acting on alternative pathways. ${ }^{47,53,59}$ Further studies are needed to comprehensively characterize the anticancer mechanisms of clinically achievable doses of obatoclax in hematological malignancies, both in cultured cells and in vivo.

Given the antiapoptotic activity of BCL-2 and related family members, studies have also explored the ability of obatoclax to overcome drug resistance in tumor cells. In hematological tumor cells, obatoclax sensitivity was maintained even upon activation of prosurvival stromal signaling. ${ }^{52,58}$ It also restored sensitivity to dexamethasone, melphalan, or rituximab in chemoresistant cells. ${ }^{50,56,58}$
Potentiation of therapeutic efficacy has also been observed in hematological tumor cells or xenografts treated with combinations of obatoclax with both traditional chemotherapeutics (eg, fludarabine, ${ }^{52,55}$ chlorambucil, ${ }^{55}$ cisplatin, ${ }^{56}$ doxorubicin, ${ }^{56}$ vincristine, ${ }^{56}$ and cytarabine ${ }^{47}$ ) and targeted therapeutics or compounds (eg, proteasome inhibitors, ${ }^{54,57,60}$ multiple kinase inhibitors, ${ }^{48,49,54,61-63}$ TRAIL, ${ }^{64}$ and histone deacetylase inhibitors ${ }^{65-67}$ ). In some cases, these therapeutic interactions occur through obatoclax-mediated suppression of antiapoptotic BCL-2 member upregulation that can occur as a feedback response to the coadministered agent. ${ }^{43,57,58,60}$ Interestingly, elevated levels of MCL-1 can confer resistance to ABT-737 in tumor cells, while a combination of ABT-737 with the more promiscuous BCL-2 family inhibitor obatoclax triggers a robust therapeutic effect. ${ }^{43,47}$

Given the activity observed in cell culture models, obatoclax was also evaluated in preclinical mouse models. A therapeutic effect of obatoclax as a single agent was observed in xenografts of cell lines derived from solid epithelial tumors or a lymphoma. ${ }^{43,68}$ Single-agent efficacy of obatoclax has not yet been reported in mouse models of other hematological malignancies. However, combining obatoclax treatment with (1) sorafenib in AML, ${ }^{62}$ (2) flavopiridol in multiple myeloma, ${ }^{63}$ or (3) the oncolytic vesicular stomatitis virus (VSV) in lymphoma ${ }^{68}$ significantly reduced xenograft tumor volume. Collectively, these studies provided important information on the potential safety and tolerability of obatoclax. Administration of obatoclax did not result in animal weight loss or other indicators of generalized toxicity, but sometimes led to neurological toxicity at high doses, ${ }^{58,62,63,69}$ foreshadowing adverse events to be noted in clinical trials.

To further probe the potential therapeutic window of this compound, effects of obatoclax on normal hematopoietic progenitors were also investigated. In general, obatoclax has demonstrated a preferential cytotoxic effect in hematological tumor cells compared to mononuclear cells from peripheral blood or bone marrow of healthy donors, or primitive progenitor-enriched $\mathrm{CD} 34^{+}$fractions thereof. ${ }^{57,58,62}$ In addition, obatoclax treatment was not myelosuppressive in xenografted mice. ${ }^{58,62}$ However, other reports have observed that obatoclax can reduce the $\mathrm{CD} 34^{+} \mathrm{CD} 38^{-}$stem cell-enriched fraction derived from either normal or leukemic cells following xenotransplantation, and prolonged exposure to obatoclax can reduce the colony-formation ability of mononuclear cells from normal bone marrow. ${ }^{58,69}$ Together, preclinical studies have therefore identified obatoclax as a putative novel anticancer agent with potential for a promising therapeutic 
index. Neurological symptoms and myelosuppression were also identified as candidate toxicities to monitor closely in clinical trials of obatoclax mesylate.

\section{Clinical evaluation of obatoclax mesylate as a single agent}

The mesylate salt of obatoclax has been formulated for intravenous (iv) administration. Obatoclax mesylate has been evaluated as a single agent in several independent Phase I or II clinical trials in patients with advanced, often heavily pretreated, hematological malignancies (Table 1). While they are beyond the scope of this review, it is notable that clinical trials of obatoclax mesylate have been completed or are ongoing in solid tumors as well, particularly in lung carcinoma. ${ }^{36,42}$ Together, these studies have provided crucial information regarding tolerable doses and treatment schedules, common drug-related adverse events, pharmacokinetics, and clinical activity in some patients.

\section{Leukemia}

O'Brien et al reported a Phase I trial treating 26 CLL patients with obatoclax mesylate. ${ }^{70}$ Doses were escalated to $40 \mathrm{mg} / \mathrm{m}^{2}$ over 3-hour infusions occurring every 3 weeks. Dose-related, transient CNS symptoms were observed upon obatoclax exposure, including somnolence, ataxia, and confusion. These toxicities were dose-limiting, with a maximum tolerated dose (MTD) of $28 \mathrm{mg} / \mathrm{m}^{2}$ over 3 hours every 3 weeks. One patient (4\%), who received the lowest dose of $3.5 \mathrm{mg} / \mathrm{m}^{2}$ of obatoclax mesylate over 1 hour every 3 weeks, achieved a partial response (PR). Several anemic or thrombocytopenic patients displayed hematological improvement, increasing hemoglobin levels, and platelet counts, and some patients achieved transfusion independence.

In another Phase I study, 44 patients with AML, ALL, CLL, or myelodysplasia were treated with obatoclax mesylate over 24-hour infusions at different doses, frequencies, and intervals to determine an optimal dose for subsequent Phase II studies. ${ }^{71}$ Infusions of up to $28 \mathrm{mg} / \mathrm{m}^{2}$ per day over a 24-hour period, for up to 4 days every 2 weeks, were welltolerated without dose-limiting toxicity. While a substantial proportion of patients experienced grade 1 or 2 symptoms related to central nervous system (CNS) activity, including somnolence or fatigue, dizziness, euphoria, and gait disturbances culminating in a feeling of inebriation, these neurological symptoms were not dose-limiting. However, further dose escalation was not recommended, given the challenges to managing any worsening of such symptoms in an outpatient setting. In this trial, one AML patient (4\%) achieved a complete response (CR) upon treatment with $20 \mathrm{mg} / \mathrm{m}^{2}$ of obatoclax mesylate over $24 \mathrm{~h}$ every week, which was maintained over eight months. Additionally, three patients with myelodysplasia (21\%) demonstrated hematological improvement, becoming transiently independent of transfusions of platelets or red blood cells.

\section{Lymphoma}

In a Phase I study of obatoclax mesylate in advanced malignancies, a PR was attained in one patient with large cell lymphoma treated with 21 to $28 \mathrm{mg} / \mathrm{m}^{2}$ over 3 hours every week..$^{72}$ This response was maintained for 2 months. An additional lymphoma patient maintained stable disease throughout 72 weeks of treatment with $7 \mathrm{mg} / \mathrm{m}^{2}$ over 3 hours every 3 weeks. Dose-limiting CNS toxicities were observed, including somnolence and abnormal coordination, leading to the recommendation of weekly administration of no more than $20 \mathrm{mg} / \mathrm{m}^{2}$ of obatoclax mesylate over 3-hour infusions in future studies. Encouraged by the response in lymphoma, a Phase II study of obatoclax mesylate in 13 pretreated, relapsed, or refractory classical Hodgkin lymphoma patients was conducted and recently reported. ${ }^{73}$ While treatment with a set dose of $60 \mathrm{mg}$ of obatoclax mesylate over 24 hours every 2 weeks was well-tolerated and several patients maintained stable disease, no PR or CR was observed.

\section{Myelofibrosis}

The efficacy of obatoclax in the treatment of myelofibrosis has also been examined in a single Phase II study. ${ }^{74}$ Twentytwo pretreated patients were administered a set dose of $60 \mathrm{mg}$ of obatoclax mesylate over 24 hours every 2 weeks, with the option of dose reduction to $45 \mathrm{mg}$ if dose-limiting toxicities were observed. These included the CNS symptom of ataxia, along with a single case of febrile neutropenia. While one patient demonstrated hematological improvement, with increases in hemoglobin and platelet counts and independence of red blood cell transfusion, no PR or CR was observed.

\section{Tolerability and pharmacokinetics}

Together, the early-phase clinical trials of obatoclax mesylate have provided a wealth of information on its safety and pharmacokinetic (PK) properties. The consistent observation of neurological symptoms in these trials has been deemed clearly drug-related, although the mechanistic basis for this finding is uncertain. These adverse events occurred shortly after infusion initiation, resolved quickly after infusion completion, and appeared 
to be dose-related. ${ }^{70,71}$ Affecting from $40 \%$ to over $90 \%$ of patients, these neurological symptoms were the most common toxicities associated with obatoclax mesylate administration..$^{70-74}$ Other relatively common observed toxicities include gastrointestinal symptoms, dyspnea, and cough. ${ }^{70-74}$ In the initial Phase I trial of obatoclax mesylate in hematological malignancies, one patient displayed a grade 3 hypersensitivity reaction to the drug. ${ }^{71}$ This was successfully managed with antihistamines and corticosteroids, which have sometimes been included as a pretreatment in subsequently treated patients..$^{71,74,75}$

Several laboratory-based adverse effects have also been observed in obatoclax mesylate-treated patients. These do not appear to be clearly dose-related and have largely been discounted as drug-specific effects. For instance, QTc prolongation was observed in electrocardiograms of some patients in early trials, but these were found to reflect preexisting conditions. ${ }^{71}$ Similarly, observed cytopenias are thought to reflect disease progression rather than a myelosuppressive effect of obatoclax, consistent with animal toxicology studies. $^{58,70-72,74}$

The clinical studies have also established several PK properties of obatoclax mesylate. While these trials have assessed PK parameters following different doses or schedules of obatoclax mesylate infusions, some consistent observations can be noted. The peak plasma concentration $\left(\mathrm{C}_{\max }\right)$ ranged from three to $16 \mathrm{ng} / \mathrm{mL}$ (7 to $39 \mathrm{nM}$ ) following 24-hour infusions, ${ }^{71,73,74}$ or 14 to $155 \mathrm{ng} / \mathrm{mL}$ ( 34 to $375 \mathrm{nM}$ ) following 3-hour infusions, ${ }^{70,72}$ generally exhibiting a dose-dependent increase. Plasma levels also tended to rapidly reach a steady state prior to infusion completion, and were similar between single day and multiday dosing schedules. ${ }^{70,71,74}$ Elimination half-life $\left(t_{1 / 2}\right)$ values varied over a range of 7 to 14 hours following 24-hour infusions, ${ }^{71}$ or 24 to 60 hours following 3 -hour infusions, ${ }^{70,72}$ although it was noted in the latter case that the majority of drug is eliminated with a short $\mathrm{t}_{1 / 2}{ }^{70}$ While the concentration of free obatoclax accumulated within tumor cells in these trials remains unknown, it is notable that some of the antitumor responses observed in preclinical studies in hematological malignancies have been detected in the nanomolar concentration range..$^{47-52,56,58}$

\section{Pharmacodynamic markers and responder hypotheses}

Taken together, obatoclax mesylate can be safely administered at doses that have signals of clinical activity. The small number of impressive objective responses introduces the major outstanding question: what differentiates responders from nonresponders?

\section{Pharmacodynamic markers}

One strategy to address heterogeneity in sensitivity to obatoclax is to identify and examine pharmacodynamic (PD) markers that can be retro- or prospectively correlated with response in clinical trials. Thus far, these efforts have been restricted to detecting molecular correlates of apoptosis in patient plasma or circulating leukemic cells.

In both the CLL trial of O'Brien et al and the trial in solid tumors and lymphomas of Hwang et al, increases in oligonucleosomal DNA:histone complex levels characteristic of apoptosis were detected in patient plasma following treatment with high doses of obatoclax mesylate. ${ }^{70,72}$ While in the former study these increases were proportional to the dose administered, this did not hold true in the latter trial, perhaps indicating differences in applicability of this PD marker or differential apoptosis-inducing activity of obatoclax in different tumor contexts. In addition, plasma levels of these complexes do not correlate solely with apoptosis; for instance, their levels may be modified by macrophage activity. ${ }^{76}$ Furthermore, this indirect measure of apoptosis cannot address the mechanism by which apoptosis was triggered. Notably, the two strongest clinical responses observed in these trials occurred in patients where an increase in plasma oligonucleosomal DNA was not detected, and therefore the association of this potential PD marker with clinical response remains unclear. ${ }^{70,72}$ Alternative approaches to assessing apoptosis in circulating hematopoietic cells have included the detection of a reduction in circulating lymphocytes in CLL, or dual Annexin V/propidium iodide (PI) staining of CLL lymphocytes in peripheral blood. ${ }^{70,77}$

As discussed, many preclinical studies suggest that obatoclax acts at least in part through modulation of BAX/ BAK-driven apoptosis. It is not yet clear, however, whether these same mechanisms of action occur in cancer patients. To evaluate such "on-target" effects of obatoclax in the clinical context, O'Brien et al detected increased levels of active BAX/BAK heterocomplexes in the peripheral blood mononuclear cells of $12 / 16(75 \%)$ obatoclax mesylate-treated CLL patients. ${ }^{70}$ This is consistent with a $\mathrm{BH} 3$ mimetic effect of obatoclax, whereby its binding to antiapoptotic BCL-2 family members would release functional BAX or BAK for homo- or heterooligomerization (Figure 1C). Integrating assays such as these into future clinical trials may clarify the correlation of BAX/BAK activation with response to obatoclax mesylate in cancer patients.

\section{Responder hypotheses}

A complementary approach to probing the mechanisms governing the response of hematological malignancies 
to obatoclax is to identify defining features of obatoclaxsensitive tumor cells. For instance, the single AML patient that achieved a robust CR had a chromosomal rearrangement at the mixed lineage leukemia (MLL) locus and was previously untreated, prompting the hypothesis that either treatment naïve and/or MLL-associated leukemia may be preferentially obatoclax-sensitive. ${ }^{71}$ The trial included three other MLL-associated AML patients that had all relapsed following induction chemotherapy, along with two other previously untreated AML patients, none of whom responded to obatoclax mesylate therapy. Thus, these hypotheses remain to be fully addressed. ${ }^{71}$ Notably, the single CLL patient that achieved a PR upon obatoclax mesylate treatment was also the only patient in the trial that was previously untreated with alkylating agents. ${ }^{70}$ These observations suggest that obatoclax may be more beneficial in less heavily pretreated patients, perhaps as a result of acquired multidrug resistance in pretreated, refractory, or relapsed patients. Phase II trials of obatoclax mesylate in untreated AML and myelodysplastic syndrome (MDS) have recently been completed, analysis of which may help address this question. ${ }^{36}$

If obatoclax acts in part as a $\mathrm{BH} 3$ mimetic in vivo, therapeutic response may also differ according to the nature of dysregulation of BCL-2 family members (recently reviewed by Davids and Letai ${ }^{33}$ ). For example, hematological tumor cells dependent on high levels of BCL-2 to bind and neutralize high levels of the proapoptotic $\mathrm{BH} 3$-only activator BIM are particularly sensitive to the BH3 mimetic ABT-737, which can compete for binding to BCL-2, releasing BIM to activate BAK ${ }^{78-80}$ Occupancy of the $\mathrm{BH} 3$ domain of antiapoptotic BCL-2 members with proapoptotic activator BH3-only members can play a significant role in how "primed" a cell is toward undergoing apoptosis, which can be assessed experimentally using peptide probes in a technique called $\mathrm{BH} 3$ profiling developed by the Letai group..$^{33,78}$ This approach has been used successfully to profile primary samples and cell lines derived from hematological malignancies to predict response to ABT-737 and chemotherapy. ${ }^{13,79-81}$ Evaluating the balance of BCL-2 family proteins either retro- or prospectively in clinical samples collected in obatoclax mesylate trials may reveal a particular profile of BCL-2 family members conferring susceptibility to obatoclax mesylate as a single agent, as well as tumors that may benefit from using it as a "priming agent" to increase overall sensitivity to other anticancer therapeutics.

Integrating generalized apoptosis-based PD markers into further clinical evaluation of obatoclax mesylate may allow responders and nonresponders to be differentiated at a molecular level. Furthermore, a more thorough understand- ing of the mechanism of obatoclax in cancer patients and molecular susceptibilities in the balance of BCL-2 family proteins may provide insight into additional molecular hallmarks of obatoclax-sensitive malignancies.

\section{Clinical evaluation of combination treatments}

Obatoclax mesylate has demonstrated limited but intriguing clinical efficacy as a single agent, and preclinical evidence suggests that its potential may be fully realized in combination with other therapeutics. To this end, several trials combining obatoclax mesylate with other agents in hematological malignancies have been reported in abstract form, and others are ongoing. These trials include three Phase I studies combining obatoclax mesylate with (1) bortezomib in mantle cell lymphoma, with $3 / 12$ patients achieving a $\mathrm{CR} ;{ }^{82}$ (2) bortezomib in multiple myeloma, with $4 / 10$ patients achieving a $\mathrm{PR} ;{ }^{75}$ and (3) fludarabine and rituximab in CLL, with an overall response rate of at least $54 \% .{ }^{77}$ Furthermore, the clinical trials registry of the US National Institutes of Health (NIH) lists two National Cancer Institute (NCI)sponsored Phase I trials of obatoclax mesylate in combination with (1) bortezomib in aggressive non-Hodgkin lymphoma, where recruitment is ongoing; and with (2) vincristine, doxorubicin, and dexrazoxane in young patients with solid or hematological malignancies, in an ongoing trial where recruitment is complete. ${ }^{36}$ Promising results from these trials may encourage future Phase II trials of obatoclax mesylate in combination treatments, where efficacy can be formally evaluated. In addition, well-designed preclinical studies may provide a rationale for novel combination treatments including obatoclax mesylate to be evaluated in subsequent Phase I trials. For example, Rahmani et al have recently provided preclinical rationale for treating AML with a combination of obatoclax and the multi-kinase inhibitor sorafenib, which induces MCL-1 downregulation. ${ }^{62}$

\section{Summary of evidence}

To date, obatoclax mesylate has been evaluated in several independent, nonrandomized early-phase clinical trials, providing level 3 evidence of its potential in the treatment of hematological malignancies. Substantial evidence indicates that obatoclax mesylate can be safely administered to patients with a variety of hematological malignancies at the doses and schedules recommended by Phase I studies. The trials discussed herein also provide limited evidence of the clinical activity of obatoclax mesylate in patients with hematological malignancies in terms of objective responses (AML, CLL, lymphoma) and hematological improvement 
(myelodysplasia, CLL, myelofibrosis). While the number of objective responses to obatoclax mesylate as a single agent has been small, formal assessment of clinical efficacy must be addressed in indication-specific Phase II trials, which would benefit from inclusion of companion studies addressing responder hypotheses. Given emerging evidence from preclinical models and trials presented in abstract form, there are also indications that obatoclax may be more effective when used in combination with other anticancer therapeutics. As more data from ongoing trials become available, optimal therapeutic indications, biomarkers of response, and rational combination treatments may be elucidated.

\section{Disclosure}

ADS is a Leukemia and Lymphoma Society Scholar in Clinical Research. The authors report no conflicts of interest in this work.

\section{References}

1. Kroemer G, Galluzzi L, Brenner C. Mitochondrial membrane permeabilization in cell death. Physiol Rev. 2007;87(1):99-163.

2. Galluzzi L, Vitale I, Abrams JM, et al. Molecular definitions of cell death subroutines: recommendations of the Nomenclature Committee on Cell Death 2012. Cell Death Differ. 2012;19(1):107-120.

3. Tait SW, Green DR. Mitochondria and cell death: outer membrane permeabilization and beyond. Nat Rev Mol Cell Biol. 2010;11(9): 621-632.

4. Happo L, Strasser A, Cory S. BH3-only proteins in apoptosis at a glance. J Cell Sci. 2012;125(Pt 5):1081-1087.

5. Tsujimoto Y, Cossman J, Jaffe E, Croce CM. Involvement of the bcl-2 gene in human follicular lymphoma. Science. 1985;228(4706): 1440-1443.

6. Pegoraro L, Palumbo A, Erikson J, et al. A 14;18 and an 8;14 chromosome translocation in a cell line derived from an acute B-cell leukemia. Proc Natl Acad Sci U S A. 1984;81(22):7166-7170.

7. McDonnell TJ, Deane N, Platt FM, et al. bcl-2-immunoglobulin transgenic mice demonstrate extended B cell survival and follicular lymphoproliferation. Cell. 1989;57(1):79-88.

8. Vaux DL, Cory S, Adams JM. Bcl-2 gene promotes haemopoietic cell survival and cooperates with c-myc to immortalize pre-B cells. Nature. 1988;335(6189):440-442.

9. Korsmeyer SJ. Bcl-2 initiates a new category of oncogenes: regulators of cell death. Blood. 1992;80(4):879-886.

10. Youle RJ, Strasser A. The BCL-2 protein family: opposing activities that mediate cell death. Nat Rev Mol Cell Biol. 2008;9(1):47-59.

11. Kitada S, Pedersen IM, Schimmer AD, Reed JC. Dysregulation of apoptosis genes in hematopoietic malignancies. Oncogene. 2002;21(21): 3459-3474.

12. Soltani-Arabshahi R, Leboeuf C, Rivet J, et al. Bcl-xL gene expression correlated with lower apoptotic cell numbers and shorter progressionfree survival in PCFCL. J Invest Dermatol. 2009;129(7):1703-1709.

13. Ni Chonghaile T, Sarosiek KA, Vo TT, et al. Pretreatment mitochondrial priming correlates with clinical response to cytotoxic chemotherapy. Science. 2011;334(6059):1129-1133.

14. Kornblau SM, Vu HT, Ruvolo P, et al. BAX and PKCalpha modulate the prognostic impact of BCL2 expression in acute myelogenous leukemia. Clin Cancer Res. 2000;6(4):1401-1409.

15. Del Poeta G, Venditti A, Del Principe MI, et al. Amount of spontaneous apoptosis detected by Bax/Bcl-2 ratio predicts outcome in acute myeloid leukemia (AML). Blood. 2003;101(6):2125-2131.
16. Tóthóva E, Fricova M, Stecová N, Kafková A, Elbertová A. High expression of Bcl-2 protein in acute myeloid leukemia cells is associated with poor response to chemotherapy. Neoplasma. 2002;49(3): 141-144.

17. Kitada S, Andersen J, Akar S, et al. Expression of apoptosis-regulating proteins in chronic lymphocytic leukemia: correlations with In vitro and In vivo chemoresponses. Blood. 1998;91(9):3379-3389.

18. Wuillème-Toumi S, Robillard N, Gomez P, et al. Mcl-1 is overexpressed in multiple myeloma and associated with relapse and shorter survival. Leukemia. 2005;19(7):1248-1252.

19. Walensky LD, Kung AL, Escher I, et al. Activation of apoptosis in vivo by a hydrocarbon-stapled BH3 helix. Science. 2004;305(5689): 1466-1470.

20. Raynaud FI, Orr RM, Goddard PM, et al. Pharmacokinetics of G3139, a phosphorothioate oligodeoxynucleotide antisense to bcl-2, after intravenous administration or continuous subcutaneous infusion to mice. J Pharmacol Exp Ther. 1997;281(1):420-427.

21. Advani PP, Paulus A, Masood A, Sher T, Chanan-Khan A. Pharmacokinetic evaluation of oblimersen sodium for the treatment of chronic lymphocytic leukemia. Expert Opin Drug Metab Toxicol. 2011;7(6):765-774.

22. O'Brien S, Moore JO, Boyd TE, et al. Randomized phase III trial of fludarabine plus cyclophosphamide with or without oblimersen sodium (Bcl-2 antisense) in patients with relapsed or refractory chronic lymphocytic leukemia. J Clin Oncol. 2007;25(9):1114-1120.

23. O'Brien S, Moore JO, Boyd TE, et al. 5-year survival in patients with relapsed or refractory chronic lymphocytic leukemia in a randomized, phase III trial of fludarabine plus cyclophosphamide with or without oblimersen. J Clin Oncol. 2009;27(31):5208-5212.

24. Limited AI. Oblimersen: Augmerosen, BCL-2 antisense oligonucleotide - Genta, G 3139, GC 3139, oblimersen sodium. Drugs R D. 2007;8(5):321-334

25. Chanan-Khan AA, Niesvizky R, Hohl RJ, et al. Phase III randomised study of dexamethasone with or without oblimersen sodium for patients with advanced multiple myeloma. Leuk Lymphoma. 2009;50(4):559-565.

26. Zhai D, Jin C, Satterthwait AC, Reed JC. Comparison of chemical inhibitors of antiapoptotic Bcl-2-family proteins. Cell Death Differ. 2006;13(8):1419-1421.

27. Tse C, Shoemaker AR, Adickes J, et al. ABT-263: a potent and orally bioavailable Bcl-2 family inhibitor. Cancer Res. 2008;68(9): 3421-3428.

28. Kitada S, Leone M, Sareth S, Zhai D, Reed JC, Pellecchia M. Discovery, characterization, and structure-activity relationships studies of proapoptotic polyphenols targeting B-cell lymphocyte/leukemia-2 proteins. J Med Chem. 2003;46(20):4259-4264.

29. Shore GC, Viallet J. Modulating the bcl-2 family of apoptosis suppressors for potential therapeutic benefit in cancer. Hematology Am Soc Hematol Educ Program. 2005:226-230.

30. Oltersdorf T, Elmore SW, Shoemaker AR, et al. An inhibitor of Bcl-2 family proteins induces regression of solid tumours. Nature. 2005;435(7042):677-681.

31. Rudin CM, Hann CL, Garon EB, et al. Phase II study of single-agent navitoclax (ABT-263) and biomarker correlates in patients with relapsed small cell lung cancer. Clin Cancer Res. 2012;18(11):3163-3169.

32. Wilson WH, O'Connor OA, Czuczman MS, et al. Navitoclax, a targeted high-affinity inhibitor of BCL-2, in lymphoid malignancies: a phase 1 dose-escalation study of safety, pharmacokinetics, pharmacodynamics, and antitumour activity. Lancet Oncol. 2010;11(12):1149-1159.

33. Davids MS, Letai A. Targeting the B-cell lymphoma/leukemia 2 family in cancer. J Clin Oncol. 2012;30(25):3127-3135.

34. Leber B, Geng F, Kale J, Andrews DW. Drugs targeting Bcl-2 family members as an emerging strategy in cancer. Expert Rev Mol Med. 2010;12:e28.

35. Souers AJ, Leverson JD, Boghaert ER, et al. ABT-199, a potent and selective BCL-2 inhibitor, achieves antitumor activity while sparing platelets. Nat Med. 2013. 
36. ClinicalTrials.gov, A Service of the US National Institutes of Health [web site on the Internet]. Available from: http://clinicaltrials.gov, Accessed December 12, 2012.

37. Heist RS, Fain J, Chinnasami B, et al. Phase I/II study of AT-101 with topotecan in relapsed and refractory small cell lung cancer. J Thorac Oncol. 2010;5(10):1637-1643.

38. Liu G, Kelly WK, Wilding G, Leopold L, Brill K, Somer B. An openlabel, multicenter, phase I/II study of single-agent AT-101 in men with castrate-resistant prostate cancer. Clin Cancer Res. 2009;15(9): 3172-3176.

39. Ready N, Karaseva NA, Orlov SV, et al. Double-blind, placebo-controlled, randomized phase 2 study of the proapoptotic agent AT-101 plus docetaxel, in second-line non-small cell lung cancer. J Thorac Oncol. 2011;6(4):781-785.

40. Baggstrom MQ, Qi Y, Koczywas M, et al. A phase II study of AT-101 (Gossypol) in chemotherapy-sensitive recurrent extensive-stage small cell lung cancer. J Thorac Oncol. 2011;6(10):1757-1760.

41. Sonpavde G, Matveev V, Burke JM, et al. Randomized phase II trial of docetaxel plus prednisone in combination with placebo or AT-101, an oral small molecule Bcl-2 family antagonist, as first-line therapy for metastatic castration-resistant prostate cancer. Ann Oncol. 2012;23(7):1803-1808.

42. Joudeh J, Claxton D. Obatoclax mesylate: pharmacology and potential for therapy of hematological neoplasms. Expert Opin Investig Drugs. 2012;21(3):363-373.

43. Nguyen M, Marcellus RC, Roulston A, et al. Small molecule obatoclax (GX15-070) antagonizes MCL-1 and overcomes MCL-1-mediated resistance to apoptosis. Proc Natl Acad Sci U S A. 2007;104(49) 19512-19517.

44. Hussain SR, Cheney CM, Johnson AJ, et al. Mcl-1 is a relevant therapeutic target in acute and chronic lymphoid malignancies: down-regulation enhances rituximab-mediated apoptosis and complement-dependent cytotoxicity. Clin Cancer Res. 2007;13(7):2144-2150.

45. Derenne S, Monia B, Dean NM, et al. Antisense strategy shows that Mcl-1 rather than Bcl-2 or Bcl-x(L) is an essential survival protein of human myeloma cells. Blood. 2002;100(1):194-199.

46. Wenzel SS, Grau M, Mavis C, et al. MCL1 is deregulated in subgroups of diffuse large B-cell lymphoma. Leukemia. 2012.

47. Konopleva M, Watt J, Contractor R, et al. Mechanisms of antileukemic activity of the novel Bcl-2 homology domain-3 mimetic GX15-070 (obatoclax). Cancer Res. 2008;68(9):3413-3420.

48. Buet D, Gallais I, Lauret E, et al. Cotargeting signaling pathways driving survival and cell cycle circumvents resistance to Kit inhibitors in leukemia. Blood. 2012;119(18):4228-4241.

49. Aichberger KJ, Gleixner KV, Mirkina I, et al. Identification of proapoptotic Bim as a tumor suppressor in neoplastic mast cells role of KIT D816V and effects of various targeted drugs. Blood. 2009;114(26):5342-5351.

50. Bonapace L, Bornhauser BC, Schmitz M, et al. Induction of autophagydependent necroptosis is required for childhood acute lymphoblastic leukemia cells to overcome glucocorticoid resistance. J Clin Invest. 2010;120(4):1310-1323.

51. Heidari N, Hicks MA, Harada H. GX15-070 (obatoclax) overcomes glucocorticoid resistance in acute lymphoblastic leukemia through induction of apoptosis and autophagy. Cell Death Dis. 2010; $1: \mathrm{e} 76$.

52. Herishanu Y, Gibellini F, Njuguna N, et al. Activation of CD44, a receptor for extracellular matrix components, protects chronic lymphocytic leukemia cells from spontaneous and drug induced apoptosis through MCL-1. Leuk Lymphoma. 2011;52(9):1758-1769.

53. Vogler M, Weber K, Dinsdale D, et al. Different forms of cell death induced by putative BCL2 inhibitors. Cell Death Differ. 2009;16(7): 1030-1039.

54. Pérez-Galán P, Roué G, López-Guerra M, et al. BCL-2 phosphorylation modulates sensitivity to the BH3 mimetic GX15-070 (Obatoclax) and reduces its synergistic interaction with bortezomib in chronic lymphocytic leukemia cells. Leukemia. 2008;22(9):1712-1720.
55. Campàs C, Cosialls AM, Barragán M, et al. Bcl-2 inhibitors induce apoptosis in chronic lymphocytic leukemia cells. Exp Hematol. 2006;34(12):1663-1669.

56. Brem EA, Thudium K, Khubchandani S, et al. Distinct cellular and therapeutic effects of obatoclax in rituximab-sensitive and -resistant lymphomas. Br J Haematol. 2011;153(5):599-611.

57. Pérez-Galán P, Roué G, Villamor N, Campo E, Colomer D. The BH3-mimetic GX15-070 synergizes with bortezomib in mantle cell lymphoma by enhancing Noxa-mediated activation of Bak. Blood. 2007;109(10):4441-4449.

58. Trudel S, Li ZH, Rauw J, Tiedemann RE, Wen XY, Stewart AK. Preclinical studies of the pan-Bcl inhibitor obatoclax (GX015-070) in multiple myeloma. Blood. 2007;109(12):5430-5438.

59. Albershardt TC, Salerni BL, Soderquist RS, et al. Multiple BH3 mimetics antagonize antiapoptotic MCL1 protein by inducing the endoplasmic reticulum stress response and up-regulating $\mathrm{BH} 3$-only protein NOXA J Biol Chem. 2011;286(28):24882-24895.

60. Dasmahapatra G, Lembersky D, Son MP, et al. Obatoclax interacts synergistically with the irreversible proteasome inhibitor carfilzomib in GC- and ABC-DLBCL cells in vitro and in vivo. Mol Cancer Ther. 2012;11(5):1122-1132.

61. López-Guerra M, Roué G, Pérez-Galán P, et al. p65 activity and ZAP70 status predict the sensitivity of chronic lymphocytic leukemia cells to the selective IkappaB kinase inhibitor BMS-345541. Clin Cancer Res. 2009;15(8):2767-2776.

62. Rahmani M, Aust MM, Attkisson E, Williams DC Jr, FerreiraGonzalez A, Grant S. Inhibition of Bcl-2 antiapoptotic members by obatoclax potently enhances sorafenib-induced apoptosis in human myeloid leukemia cells through a Bim-dependent process. Blood. 2012;119(25):6089-6098.

63. Chen S, Dai Y, Pei XY, et al. CDK inhibitors upregulate BH3-only proteins to sensitize human myeloma cells to $\mathrm{BH} 3$ mimetic therapies. Cancer Res. 2012;72(16):4225-4237.

64. Martínez-Paniagua MA, Baritaki S, Huerta-Yepez S, et al. Mcl-1 and YY1 inhibition and induction of DR5 by the BH3-mimetic Obatoclax (GX15-070) contribute in the sensitization of B-NHL cells to TRAIL apoptosis. Cell Cycle. 2011;10(16):2792-2805.

65. Jóna A, Khaskhely N, Buglio D, et al. The histone deacetylase inhibitor entinostat (SNDX-275) induces apoptosis in Hodgkin lymphoma cells and synergizes with Bcl-2 family inhibitors. Exp Hematol. 2011;39(10):1007-1017. e1.

66. Wei Y, Kadia T, Tong W, et al. The combination of a histone deacetylase inhibitor with the BH3-mimetic GX15-070 has synergistic antileukemia activity by activating both apoptosis and autophagy. Autophagy. 2010;6(7):976-978.

67. Wei Y, Kadia T, Tong W, et al. The combination of a histone deacetylase inhibitor with the Bcl-2 homology domain-3 mimetic GX15-070 has synergistic antileukemia activity by activating both apoptosis and autophagy. Clin Cancer Res. 2010;16(15):3923-3932.

68. Samuel S, Tumilasci VF, Oliere S, et al. VSV oncolysis in combination with the BCL-2 inhibitor obatoclax overcomes apoptosis resistance in chronic lymphocytic leukemia. Mol Ther. 2010;18(12):2094-2103.

69. Campbell CJ, Lee JB, Levadoux-Martin M, et al. The human stem cell hierarchy is defined by a functional dependence on Mcl-1 for selfrenewal capacity. Blood. 2010;116(9):1433-1442.

70. O'Brien SM, Claxton DF, Crump M, et al. Phase I study of obatoclax mesylate (GX15-070), a small molecule pan-Bcl-2 family antagonist, in patients with advanced chronic lymphocytic leukemia. Blood. 2009;113(2):299-305.

71. Schimmer AD, O'Brien S, Kantarjian H, et al. A phase I study of the pan bcl-2 family inhibitor obatoclax mesylate in patients with advanced hematologic malignancies. Clin Cancer Res. 2008;14(24): 8295-8301.

72. Hwang JJ, Kuruvilla J, Mendelson D, et al. Phase I dose finding studies of obatoclax (GX15-070), a small molecule pan-BCL-2 family antagonist, in patients with advanced solid tumors or lymphoma. Clin Cancer Res. 2010;16(15):4038-4045. 
73. Oki Y, Copeland A, Hagemeister F, et al. Experience with obatoclax mesylate (GX15-070), a small molecule pan-Bcl-2 family antagonist in patients with relapsed or refractory classical Hodgkin lymphoma. Blood. 2012;119(9):2171-2172.

74. Parikh SA, Kantarjian H, Schimmer A, et al. Phase II study of obatoclax mesylate (GX15-070), a small-molecule BCL-2 family antagonist, for patients with myelofibrosis. Clin Lymphoma Myeloma Leuk. 2010;10(4):285-289.

75. Stewart AK, Trudel S, Zonder JA, et al. Phase I trial of obatoclax mesylate in combination with bortezomib for treatment of relapsed multiple myeloma. J Clin Oncol. 2012;30(Suppl 15):Abstract 8013.

76. Jiang N, Reich CF 3rd, Pisetsky DS. Role of macrophages in the generation of circulating blood nucleosomes from dead and dying cells. Blood. 2003;102(6):2243-2250.

77. Brown JR, Tesar B, Werner L, et al. Obatoclax in combination with fludarabine and rituximab (FR) is well-tolerated and shows promising clinical activity in relapsed CLL/SLL. Blood (ASH Annual Meeting Abstracts). 2011;118(21):Abstract 2865.
78. Certo M, Del Gaizo Moore V, Nishino M, et al. Mitochondria primed by death signals determine cellular addiction to antiapoptotic BCL-2 family members. Cancer Cell. 2006;9(5):351-365.

79. Del Gaizo Moore V, Brown JR, Certo M, Love TM, Novina CD, Letai A. Chronic lymphocytic leukemia requires BCL2 to sequester prodeath BIM, explaining sensitivity to BCL2 antagonist ABT-737. J Clin Invest. 2007;117(1):112-121.

80. Deng J, Carlson N, Takeyama K, Dal Cin P, Shipp M, Letai A. BH3 profiling identifies three distinct classes of apoptotic blocks to predict response to ABT-737 and conventional chemotherapeutic agents. Cancer Cell. 2007;12(2):171-185.

81. Vo TT, Ryan J, Carrasco R, et al. Relative mitochondrial priming of myeloblasts and normal HSCs determines chemotherapeutic success in AML. Cell. 2012;151(2):344-355.

82. Goy AG, Ford P, Feldman T, et al. A phase I trial of the pan Bcl-2 family inhibitor obatoclax mesylate (GX15-070) in combination with bortezomib in patients with relapsed/refractory mantle cell lymphoma. Blood (ASH Annual Meeting Abstracts). 2007;110(11):Abstract 2569.
Core Evidence

\section{Publish your work in this journal}

Core Evidence is an international, peer-reviewed open-access journal evaluating the evidence underlying the potential place in therapy of drugs throughout their development lifecycle from preclinical to postlaunch. The focus of each review is to evaluate the case for a new drug or class in outcome terms in specific indications and patient groups.

\section{Dovepress}

The manuscript management system is completely online and includes a very quick and fair peer-review system, which is all easy to use. Visit http://www.dovepress.com/testimonials.php to read real quotes from published authors. 